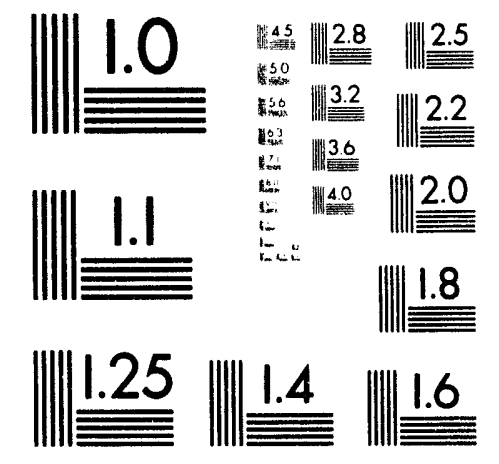



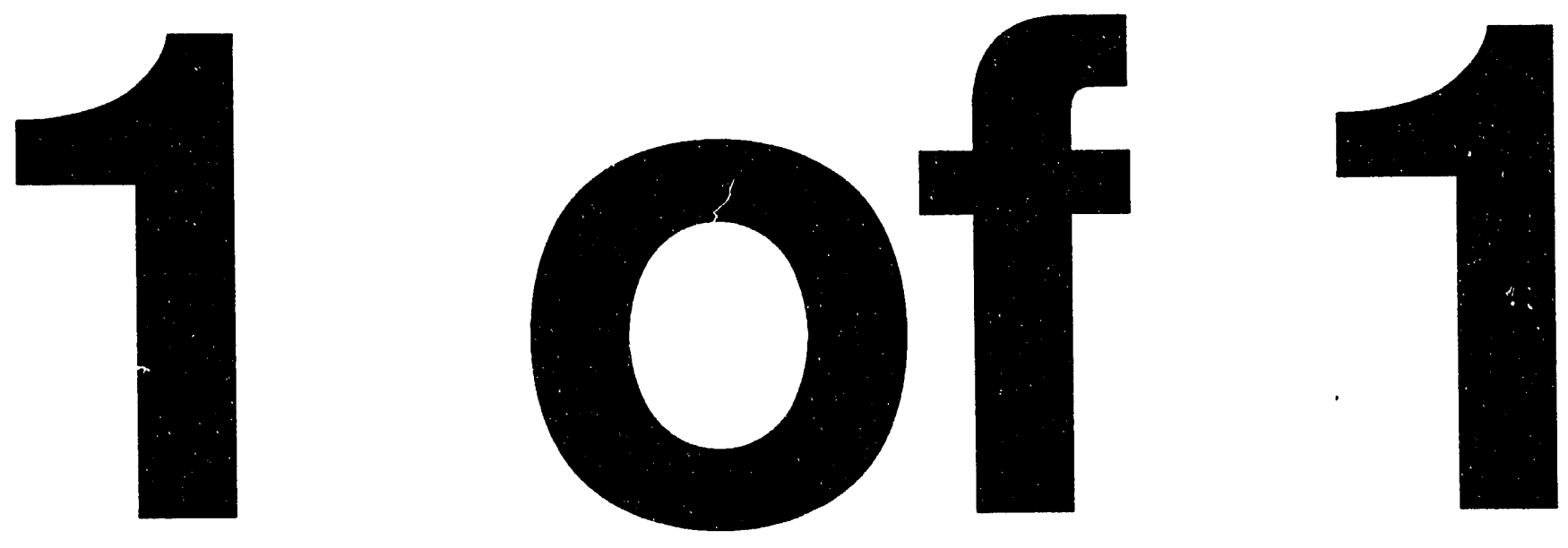


\section{ANNUAL REPORT}

\section{SCIENCE AND TECHNOLOGY ALLIANCE WORK SCHEDULE FY 1992}

\section{Institution: New Mexico Highlands University}

\section{Program Activity}

1. Program Aaministration
A. Coordinator $(10 / 1-08 / 10)$
$(08 / 10-09 / 30)$
B. Grants and Contracts

2. Faculty Development

$$
\text { A. Faculty Travel }
$$

3. Student Development

A. Student Support

B. Student Placement at Labs

4. Special Projects

A. Research and Sponsored Proj

B. Faculty Research

$$
\text { 1. Ceramics Research Project }
$$

2. Bioengineering Project (NASA JOVE)

3. Optical Crystals (NASA JOVE)

4. Chemistry Sabbatical

C. Student Research (JOVE)

D. Science Education Resource Center

E. Computer/Audio-Visual Center 1. Supervision of Center

2. Equipment

F. Physics Lab Equipment

G. Waste Management Technical Workshop

5. Curriculum Development

A. Engineering

B. Environmental Science

C. Physical Sciences

D. Biology

\section{Leader}

Dr. G. Rivera

Dr. M. McClanahan

Dr. G. Rivera

Dr. R. Clark

Dr. M. HcClanahan

Dr. M. McClanahan

Dr. M. McClanahan

Dr. R. Clark

Dr. R. Clark

Dr. L. Sveum

Dr. M. Krebs

Dr. H. W. Taylor

Dr. R. Clark

Dr. G. Sprenger

Dr. H. W. Taylor

Dr. T. Salazar

Dr. N. Illclanahan

Mr. C. Sollonub

Mr. C. Sollohub

Dr. M. McClanahan

Dr. M. McClanahan

Dr. L. Sveum

Dr. M. HeClanahan

Dr. H. McClanahan

Dr. K. Jensen

$\mathrm{Dr}$. R. Lessard

Dr. L. Sveum

Ms. N. Kirkpatrick

6. Equipment

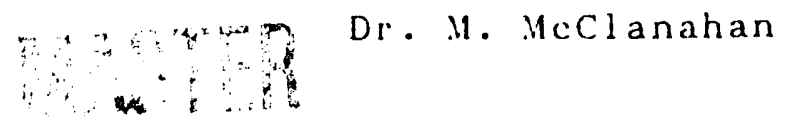




\section{SCIENCE AND TECHNOLOGY ALLIANCE MILESTONE SCHEDULE \\ FY 1992}

Institution: New Mexico Highlands University

1st Quarter. FY92

4.D. Nor 91

4.E. Fall 91

4.E. Oct 91

t.F. Fal1 91

4.G. Fall 91

5.A. Fall 91

5.B. Fall 91

5.C. Fall 91

2nd Quarter, FY92

3.A. Spring 92

3.A. Spring 92

3.B. Feb 92

4.B. March 92
Descriptive Title

Conduct SWOOPE

workshop

Purchase equipment

for Computer/AV

Center

Hire student help

for computer lab

Purchase equipment

for physics labs

Determine theme of

Fall 92 workshop

Begin curriculum

development for

Eng ineer ing

Begin curriculum revision in Env $\mathrm{Sci}$

Begin curriculum

development in

Physics

Descriptive Title

Arrange Puerto Rico trip to Waste $\mathrm{Mgt}$ conference

Supervise student poster competition

at Puerto Rico

conference

Arrange summer

placement of

students at labs

Renew contract for optical crystals grant

\section{Leader}

Dr. R. Lessard

Mr. C. Sollohub

Dr. M. McClanahan

Mr. C. Sollohub

Dr. M. McClanahan

Dr. M. MeClanahan

Dr. K. Jensen

Dr. R. Lessard

Dr. L. Sveum

Dr. D. Ross

\section{Leader}

Dr. M. HeClanahan

Dr. R. Lessard

Dr. R. Clark

Dr. M. McClanahan

Dr. R. Clark 


\begin{tabular}{|c|c|c|c|c|}
\hline 4.C. & Spring & 92 & $\begin{array}{l}\text { Select NASA student } \\
\text { scholarship awardees }\end{array}$ & Dr. H. W. Taylor \\
\hline 4.D. & Spring & 92 & $\begin{array}{l}\text { Coordinate Regional } \\
\text { Science Fair }\end{array}$ & Dr. T. Salazar \\
\hline 4.E. & Spring & 92 & $\begin{array}{l}\text { Purchase Computer / } \\
\text { AV Center equipment }\end{array}$ & $\begin{array}{l}\text { Mr. C. Sollohub } \\
\text { Dr. M. McClanahan }\end{array}$ \\
\hline 4.G. & Spring & 92 & $\begin{array}{l}\text { Complete preliminary } \\
\text { planning for Waste } \\
\text { Management Technical } \\
\text { Workshop }\end{array}$ & Dr. M. McClanahan \\
\hline S.A. & Spring & 92 & $\begin{array}{l}\text { Prepare proposal } \\
\text { for Engineering } \\
\text { program }\end{array}$ & Dr. K. Jensen \\
\hline 5. B. & Spring & 92 & $\begin{array}{l}\text { Prepare proposal } \\
\text { for revision of } \\
\text { Env Sci curriculum }\end{array}$ & Dr. R. Lessard \\
\hline 5.C. & March & 92 & $\begin{array}{l}\text { Advertise for } \\
\text { Physics faculty } \\
\text { position }\end{array}$ & Dr. L. Sveum \\
\hline 5.D. & Spring & 92 & $\begin{array}{l}\text { Teach biotechnology } \\
\text { course }\end{array}$ & Ms. N. Kirkpatrick \\
\hline 6 . & Spring & 92 & $\begin{array}{l}\text { Begin purchase of } \\
\text { equipment }\end{array}$ & Dr. M. McClanahan \\
\hline
\end{tabular}

\section{3rd Quarter. FY92}

2.A. April 32

3.A.

3.B. May 92

4.D. Spring 92

4.D. May 92

4.G. Summer 92

\section{Descriptive Title}

Attend Waste $\mathrm{Mg} t$

Conference in $P R$

Send students and

faculty to national

$\mathrm{labs}$

Develop SERC

activities for $K-12$

students/teachers

Conduct SWOOPE

workshop

Finalize plans

for Waste Management

Technical Workshop

\section{Leader}

Dr. R. Lessard

Dr. R. Clark

Dr. M. McClanahan

Dr. T. Salazar

Dr. R. Lessard

Dr. M. McClanahan 


\begin{tabular}{|c|c|c|c|c|}
\hline $5 . \lambda$. & May & 92 & $\begin{array}{l}\text { Present Engineering } \\
\text { program to } B \text { of } R \\
\text { for approval }\end{array}$ & $\begin{array}{l}\text { Dr. K. Jensen } \\
\text { Dr. M. McClanahan }\end{array}$ \\
\hline 4 th & arte & r. FY92 & Descriptive Title & Leader \\
\hline $4 . \mathrm{G}$. & Sep & 92 & $\begin{array}{l}\text { Host Waste Management } \\
\text { Technical Workshop }\end{array}$ & $\mathrm{Dr} \cdot \mathrm{M} \cdot \mathrm{McCl}$ anahan \\
\hline 5.A. & Aug & 92 & $\begin{array}{l}\text { Initiate Engineering } \\
\text { degree program }\end{array}$ & $\begin{array}{l}\text { Dr. K. Jensen } \\
\text { Dr. M. McClanahan }\end{array}$ \\
\hline $5 . B$. & Sep & 92 & $\begin{array}{l}\text { Prepare Env Sci } \\
\text { program for School } \\
\text { Curriculum approval }\end{array}$ & $\begin{array}{ll}\text { Dr. R. Lessard } \\
\text { Dr. M. McClanahan }\end{array}$ \\
\hline 6 . & Sep & 92 & $\begin{array}{l}\text { Complete equipment } \\
\text { purchases }\end{array}$ & Dr. M. McClanahan \\
\hline
\end{tabular}

Budget info removed 


\section{SCIENCE AND TECHNOLOGY ALLIANCE TECHNICAL STATUS REPORT ANNUAL REPORT FY 1992}

\section{Institution: New Mexico Highlands University}

\section{Administration}

The Science and Technology Alliance program is administered by the Vice President for Academic Affairs. Dr. Gilbert Rivera. Dr. Melinda McClanahan. the Dean of the School of Science and Engineering, served as Coordinator from 1 October 91 through 10 August 92 at which time Dr. Rivera assumed this position. The Coordinator of Research and Sponsored Projects. Dr. Ron Clark, and the Director of the Science Education Resource Center, Dr. Tomas Salazar. participate in various aspects of the project.

\section{Faculty Development}

Faculty development continues to focus on faculty travel to professional meetings and on special activities that promote the university or other program areas. One faculty member held a summer appointment at ORNL.

\section{Student Development} at S:VL.

Sixteen students held summer appointments at LANl and

\section{Special Projects}

Several special projects were completed in FYg2 or are ongoing.

A. Dr. Ron Clark is serving as the Coordinator of the Office of Research and Sponsored Projects $(1 / 4$ time).

B.1. Dr. Ron Clark, Dr. Larry Sveum. and Dr. Wi cheal Krebs are continiling research in the area of ceramics.

B.2./ Dr. H. W. Taylor and Dr. Ron Clark are continuing

B.3. work on VASA-sponsored research projects.

B.t. Dr. Cieorge Sprenger completed a one-year research sabbatical at LANiL.

E. The Computer/AV Center received heavy use by both students and faculty. 
G. The Waste Management Technical Workshop was held on the II irhlands campus september 17-19, 1992.

The workshop, entitled Research Partnerships in Hinurity lnstitutions, was co-hosted by NHHL and LAVL and attended by 82 participants from numerous ininority and majority universities, state and federal agencies, private business, and national laboratories (see Addendum 1, FY92 Fourth Quarter).

\section{Curriculum Development}

Several faculty received partial Alliance support for curriculum development in their respective areas:

\author{
Dr. Tom Cheavens \\ Dr. Dick Greene \\ Dr. Elmer Grubbs \\ Ms. Nancy Kirkpatrick \\ $\mathrm{Mr}$. Albert Maez \\ Dr. Dan Ross \\ Dr. Harry W. Taylor \\ Dr. Georige Zrilic
}

Chemistry Eng ineering Eng ineering Life Sciences

Eng ineer ing

Engineering

Engineer ing

Engr ineer ing

\section{A. Electrical Engineering Program}

The NimHU Board of Regents approved the new B.S. derree program in Electrical Engineering presented to them by Dr. Gilbert Rivera, Vice President for Academic Affairs, and Dr. Melinda $\mathrm{McCl}$ anahan. Dean of the School of Science and Technology, on May 16. 1902. The new program was initiated in the Fall 92 semester. Support of this program will be the first priority of the NuHL Alliance effort in FY93.

B. Environmental Waste hanagoment Prorram

Work continued on curriculum development for the revised B.S. degree program in Environmental Science.

C. Physical Sciences Curriculum Development

Planning continued in the development of new courses in Chemistry, Physics, and Geolory/Hydrology to support the revised Environmental Science program and in a separate Chemistry lab for Engineering and Ensineering Technology students.

The search for a new Physics faculty member was unsuccessful. and the search will be re-opened in November 1992 . 


\section{Life Sciences Curriculum Development}

A new biotechnology course was developed in Spring 91 with the help of a grant from the Department of Education's Minority Science Improvement Program (MSIP) and was taught for the second time in Spring 92. A total of 14 students have taken the course which covers several aspects of biotechnology including industrial microbiology, plant and animal cell culture, protein engineering, genetic engineering, bioremediation, and ethics. Laboratory studies include recombinant DNA techniques, organelle isolation, protoplast isolation, plant tissue culture, and SDS polyacrylamide gel electrophoresis for proteins.

\section{Equipment}

Equipment purchases included computer equipment (and software), VCR/TV monitors, environmental science field equipment, and an ultra-low temperature freezer to support the biotechnology course. 


\author{
ADDENDUM 1 \\ WASTE MANAGEMENT TECHNICAL WORKSHOP: RESEARCH PARTNERSHIPS \\ AT MINORITY INSTITUTIONS \\ NEW MEXICO HIGHLANDS UN IVERS ITY \\ 17-19 SEPTEMBER 92 \\ SPONSORED BY THE SCIENCE AND TECHNOLOGY ALLIANCE \\ U.S. DEPARTMENT OF ENERGY \\ NEW MEXICO HIGHLANDS UNIVERS ITY \\ MELINDA L . MCCLANAHAN, WORKSHOP DIRECTOR \\ LOS ALAMOS NATIONAL LABORATCRY \\ PHIL VERGAMINI, WORKSHOP COORDINATOR \\ ABAD SANDOVAL, S\&T ALLIANCE COORDINATOR
}

PREPARED BY MEL INDA L MCCLANAHAN

2 NOVEMBER 92 


\section{TABLE OF CONTENTS}

I. Narrative 1

II. Enclosures

1. List of Participants 4

2. List of Organizations 10

3. Workshop Program 11

4. Pre-Proposals/Reports from 19

5. Budget 29

6. Summary of Ratings from $34 \quad 30$ Evaluation Forms 


\section{WASTE MANAGEMENT TECHNICAL WORKSHOP: RESEARCH PARTNERSHIPS AT MINORITY INSTITUTIONS}

The Waste Management Technical Workshop: Research Partnerships at Minority Institutions was held at New Mexico Highlands University on September 17-19, 1992. This workshop was sponsored by the Department of Energy through the Science and Technology Alliance and was co-hosted by New hexico Highlands Liniversity and Los National Laboratory. The 82 participants at the first day's activitjes were from $2 j$ organizations and included scientists from Los Alamos National laboratory. Sandia Sational laboratories, and Oa's Ridnc iational Laboratory; facdly members. administrators, and sadents trom 16 universities: representatives from Fivate and conporate business; and agents from the City of Las veras. the state of yew Mexico, the l.s. Department of Energy. and the L.S. Environmental protection Agency

(Enclosures 1 and 2). Approximately b0 at tondee: pa: icipated in the last two days' activities which included a tour of solected facilities at Los Alamos Yational Laborator: and preliminary preparation of collaborative research proposals by small break-out roups. The workshop organization and manarement team consistod of Nelinda L. McClanahan, NMHC Director: Phil Vergamini. LAvit. Workshop Coordinator: Abarl Sandoval, LANL S\&TA Coordinator: and : ilppori staff (Enclosure 3, p. 17). The eight Group Pasilitators for the break-out sessions included two faculty 
members from New Mexico Highlands Lniversity, one from North Carolina AE' Lnivorsity, one staft member from Los Alamos Vational Laboratory, one from Oak Ridge National Laboratory. and three "iaculty members from non-Alliance universities (Enclosure 3, p. 16). The featured speakers and the workshop agenda are described in the program (Enclosura 3, pp. 12-15). We expect continued productive results from this :yorkizhop in the form of collaborative research projects and other cooprative efforts by both Alliance members and nonAliance attendes. To date, four research proposals have besn received by Alliance national laboratories as a direct result of this workshop. Enclosure 4 is a compilation of the pre-proposals/reports deyeloped by the eirht sroups.

This onleavor was an example of cost eflective expenditure of federal funds. Both NMHC and LANL contributed 310.000 "rom their 1092 Al liance budgets for a total of $\$ 20.000$ federal funds to finance the workshop. In addition. 00.750 in rosiatration fees was rocoived. Because of excellent $f$ iscal maracement and cost control by the ruHU Director ard her Administrative Assiztant. Ms. Cindy Griego. the expenditures for the workshop totaled onl $y: 18,197$, coisiderably under budget (Enclosure 5).

The workshop was well-planned, well-organized, and the activities finctioned smoothly. Writton evaluations from 34 participarts at the close of the workshop showed a very pusitive response to the format, the activities, and the results (Erclosure 6). The social activities were 
complimented for enhancing cameraderie and the development of new friendships. Valuable contacts were made by scientists and educators representing minority insitutions, and networking was strongly promoted. One aspect of the workshop that respondees felt could have been improved was the participation by federal agencies for the duration of the workshop.

An overall final analysis of the workshop's results is continuing, but indications are that the workshop's goals were met. We were successful, therefore, in demonstrating the beneficial impact that a small state-supported minority university such as New Mexico Highlands University can have on generating cooperative environmental research at the national level, given the active partnership with Los Alamos National Laboratory and the support of the other S\&T Alliance members and the U.S. Department of Energy. 


\section{PARTICIPANTS (NAMES/ADDRESSES) \\ WASTE MANAGEMENT TECHNICAL WORKSHOP: Research Partnerships at Minority Institutions}

Dr. Edward D. Aebischer

Oak Ridge National Laboratory

Martin Marietta Energy Systems, Inc.

P.O. Box 2008

Oak Ridge, TN 37831-6250

(615)574-7717 OFFICE

(615)576-9496 FAX

Dr. Sunnie-A Aburime

Alabama A\&M University

P.O. Box 1208

Normal, Al 35762

(205)851-5462 OFFICE

(205)851-5594 FAX

Dr. Jack Ainsworth

Rocky Mountain College

1511 Poly Dr.

Billings, MT 59102

(406)657-1101 OFFICE

(406)259-9751 FAX

Ms. Serese Aranha, Student

North Carolina A\&T State University

Greensboro, NC 27411

(919)334-7654

(919)334-7094 FAX

Mr. Federico Asmar

University of Turabo

P.O. Box 3030

Gurabo, Pueno Rico 006758

(809)743-7979 ext. 4113 OFFICE

(809)744-5476 FAX

Dr. Peter Benson

Renewable Energy Systems, Inc.

12115 So. 87th Ave.

Palos Park, IL 60464

(708) $448-1071$
Dr. Ron Bhada

Dir., Wste-Mgmt. Educ. \& Res. Ctr.

Box 30001 , Dept. 3805

New Mexico State University

Las Cruces, NM 88003-0001 646-2415

(505)646-2038 OFFICE

(505)646-4149 FAX

Dr. Kenneth P. Bentson

Environmental Science Dept.

New Mexico Highlands University

Las Vegas, NM 87701

(505)454-3501 OFFICE

(505)454-3103 FAX

Dr. Harry Boston

Oak Ridge National Laboratory

P.O. Box 2008, MS 6351

Oak Ridge, TN 37831-6351

(615)574-7840 OFFICE

(615)576-९496 FAX

Dr. Shoo-Yuh Chang

North Carolina A\&T State University

Greensboro, NC 27411

(919)334-7737 OFFICE

(919)334-7667 FAX

Dr. Eileen Chant

School of Engineering

Box 3030

Turabo University

Gurabo, Puerto Rico 007708

(809)743-7979 ext. 4143

(809)744-5476 FAX 
Dr. William R. Dawes

Sandia National Laboratories

P.O. Box 5800-Org.3500

Albuquerque, NM 87185

Dean, Sctiool of Engineering

University of Turabo

P.O. Box 3030, University Station

Gurabo, Puerto Rico 00778

(505)845-8714 OFFICE

(505)844-8884 FAX

Dr. Vasil Diyamandogiu

City College of New York

Dept. of Civil Engineering

New York, NY 10031

(212)650-8010 OFFICE

(212)650-6965 FAX

Dr. Lin Ferrand

City College of New York

Dept. of Civil Engineering $(Y-120)$

New York, NY 10031

(212)650-8017 OFFICE

(212)650-6965 FAX

Mr. Benito Garcia

Bureau Chief

Hazardous \& Radioactive Materials Bureau NMED

P.O. Box 26110

525 Camino De Los Marquez

Santa Fe, NM 87502

(505)827-4354 OFFICE

(505)827-4361 FAX

Dr. Doris Garcia

Dean of Science

F.O. Box 3030

University of Turabo

Gurabo, Puerio Rico 00778

(819)743-7979 ext. 4113 OFFICE

(819)744-5427 FAX
Ms. Karla K. Garcia

Lab/Hazardous Waste and Safety Manager

New Mexico Highlands University

(505)454-3500 OFFICE

(505) 454-3103 FAX

Dr. Piara S. Gill

Chemistry Dept., Armstrong Hall

Tuskegee University

Tuskegee, AL 36088

(205)727-8875 OFFICE

(2C5)727-7626 FAX

Dr. Ernest Gladney

Director, Environmental Science Ed.

Northem Arizona University

P.O. Box 4085

Flagstaff, AZ $86011-4085$

(602) $523-4340$ OFFICE

$(602) 523-4230$ FAX

Dr. Leo S. Gomez

SMTS, ORG 35B

Sandia National Labs

Albuquerque, NM 87175

(505)845-9764 OFFICE

(505)844-1723 FAX

Dr. Ray Graham

Rocky Mountain College

1511 Poly Dr.

Billings, MT 59102

(406)259-0589 OFFICE

(406)259-9751 FAX

Dr. Milford Greene

Director of Engineering Programs

Morehouse College

830 Wesiview Dr., S.W.

Atlanta, GA 30314-3799

(404)525-6272 OFFICE

(404)659-6536 FAX 
Dr. Alan Gutjahr

VP for Research and Economic Development New Mexico Institute of Mining and Technology

Socorro, NM

(505)835-5646 OFFICE

(505)835-5649 FAX

Dr. Merritt Helvenston

New Mexico Highlands University

Physical Sciences Department

Las Vegas, NM 87701

505-454-3244 OFFICE

505-454-0026 FAX

Dr. Constance Hill

Chairperson, Department of Geology

Box 9202

Virginia State University

Petersburg, VA 23803

(804)524-5461 OFFICE

(804)524-5738 FAX

Dr. Gerald D. Hill

Program Coordinator

Civil and Environmental Engineering Tech.

Texas Southem University

3100 Clebume

Houston, TX 77004

(713)527-7962 OFFICE

(713)639-1853 FAX

Mr. Ken Holley

S\&T Alliance Coordinator

Sandia National Laboratories

P.O. Box 5800

Albuquerque, NM 87125

(505)845-8714 OFFICE

(505)844-8884 FAX

Dr. Henry O. Hooper

Associate Vice Pres. for Academic Affairs

Box 4085

Northem Arizona University

Fiagstaff, AZ 86001

(602)523-4340 OFFICE

(602)523-4230 FAX
Ms. Pat Hurley

Science Director

Salish Kootenai College

P.O. Box 5

Ravalli, MT 59863

(406)675-4800 ext. 344 OFFICE

(406)675-4801 FAX

Dr. Gerald Z. Jacobi

Life Sciences Department

New Mexico Highlands University

Las Vegas, NM 87701

(505) 454-3412 DFFICE

(505)454-3103 FAX

Dr. Jeanette Jones

Vice President for Research \& Development

Alabama A\&M University

P.O. Box 411

Normal, AL 35762

(205)851-5675 OFFICE

(205)851-5030 FAX

Dr. James A. Kearns

University of Turabo

Box 3030

Gurabo, Puerto Rico 00778

(819)743-7979 ext. 4147 OFFICE

(819)744-5476 FAX

Dr. Jaya Krishnagopalan

Dept. of Chemical Engineering

Tuskegee University

Tuskegee, AL 36088

(205)727-8795 OFFICE

(205)727-8090 FAX

Mr. Randall Leathers, Student

North Carolina A\&T State University

Greensboro. NC 27411

(919)274-4815 OFFICE

(919)334-7094 FAX 
Dr. Dorothy Leflore

North Carolina A\&T State University

1601 E. Market St., Suite 400

Greensboro, NC 27411

(919)334-7654 OFFICE

(919)334-7094 FAX

Dr. Robert Lessard

Physical Sciences Department

New Mexico Highlands University

Las Vegas, NM 87701

505-454-3505 OFFICE

505-454-3103 FAX

Ms. Amy Long

North Carolina A\&T State University

Greensboro, NC 27441

(919)334-7654 OFFICE

(919)334-7904 FAX

Mr. Lindsay Lovejoy

State Attorney's Office

Bataan Memorial Bldg.

Santa Fe, NM 87501

(505)827-6695 OFFICE

(505)827-5826 FAX

Ms. Barbara Lynch (Student)

Southern University

3030 Congress Bldg., \#46

Baton Rouge, LA 70813

(504)771-5870 OFFICE

(504)771-4722 FAX

Ms. Jill Lytle

Deputy Asst. Sec.

Office of Waste Management

U.S. Deparment of Energy

Washington, D.C.

(202)586-0370 OFFICE

(202)586-0449 FAX
Dr. Melinda L. McClanahan

Dean, School of Science \& Engineering

New Mexico Highlands University

Las Vegas, NM 87701

(505)454-3259 OFFICE

(505)454-3103 FAX

Dr. Guillemo Martinez

University of Turabo

P.O. Box 3030

Gurabo, Puerto Rico 006758

(809)743-7979 ext. 4113 OFFICE

(809)744-5476 FAX

Dr. Wondi Mersie

Agricultural Research Station

Dept. of Lije Sciences

Virginia State University

Petersburg, VA 23803

(804)524-5652 OFFICE

(804)524-5738 FAX

Dr. Warren F. Miller,

Mail Stop A-104

Associate Director for Research and Educatior.

Los Alamos $\mathrm{Na}^{+i}$ nnal Laboratory

Los Alamos, NM 87545

(505)667-7978 OFFICE

(505)665-6163 FAX

Dr. Charles T. Mitchell

Environmental Protection Agency

Washington, D.C.

(202)260-7448 OFFICE

(202)260-4524 FAX

Mr. Les Montoya

City of Las Vegas

1700 N. Grand Ave.

Las Vegas, NM 87701

(505)454-1401 
Mr. David Moss

Los Alamos National Laboratory

Section Leader

Los Alamos, NM 87545

(505)665-3750 OFFICE

(505)667-? FAX

Dr. Stuart A. Nicholson

New Mexico Highlands University

Las Vegas, NM 87701

(505)454-3176 OFFICE

(505) 454-3103 FAX

Dr. Fred Norwood

Division 1432

Sandia National Laboratories

P.O. Box 5800

Albuquerque, NM 87175

(505)845-8508 OFFICE

(505)844-0918 FAX

Dr. Wilbert Odem

Northern Arizona University

P.O. Box 4085

Flagstaff, AZ 86011-4085

(602)523-4340 OFFICE

(602)523-4230 FAX

Dr. Chukwu Onu

Southern University and A\&M College

Baton Rouge, LA 70813

(509)376-6371 OFFICE

(509)376-1867 FAX

Ms.Catherine Ratliff

North Carolina A\&T State University

Greensboro, NC 27411

(919)334-7E54 OFFICE

(919)334-7904 FAX

Dr. Edgard Resto

University of Turabo

P.O. Box 3030

Gurabo, Puero Rico 006758

(809)743-7979 ext. 4113 OFFICE

(809) $744-5427$ FAX
Dr. Chet Richmond

Oak Ridge National Laboratory

Martin Marietta Systems, Inc.

Oak Ridge, TN 37831-6469

(615)576-3886 OFFICE

(615)576-9496 FAX

Dr. John P. Rier

Howard University

Washington, D.C. 20059

(202)806-6939 OFFICE

(202)806-4562 FAX

Dr. Agustin Rios

University of Turabo

P.O. Box 3030

Gurabo, Puerto Rico 006758

(809)743-7979 ext. 4113 OFFICE

(809)744-5476 FAX

Dr. David Sanchez

Deputy Assoc. Dir. for Research and Educ.

Los Alamos National Laboratory

Los Alamos, NM 87545

(505)667-?

(505)665-5596

Mr. Abad Sandoval

Mail Stop P355

Los Alamos National Lab

Los Alamos, NM 87545

(505)667-1230 OFFICE

(505)665-5596 FAX

Dr. Dilip T. Shah

North Carolina A\&T State University

Greensboro, NC 27411

(919)334-7586 OFFICE

(919)334-7098 FAX

Dr. J.P. Shipley

Los Alamos National Laboratory

P.O. Box Udo3, MSF641

Los Alamos, NM 87545

(505)667-2211 OFFICE

(505)665-8190 FAX 
Dr. Kenneth G. Soderstrom

School of Engineering

University of Turabo

Gurabo, Puerto Rico 00778

(809)743-7979 ext. 4113 OFFICE

(809)744-5476 FAX

Dr. Ethel F. Taylor

North Carolina A\&T State University

Greensboro, NC 27411

(919)334-7.654 OFFICE

(919)334-7094 FAX

Dr. Robert W. Taylor

Alabama A\&M University

P.O. Box 1208

Normal, AL 35762

(205)851-5462 OFFICE

(205)851-5594 FAX

Dr. Jaiara S. Turay

St. Augustine's College

Raleigin, NC 27610-2298

(Q19)828-4451 ext. 243 OFFICE

(Q19)828-0817 FAX

Dr. John Uribe

Business Department

New Mexico Highlands University

Las Vegas, NM 87701

(505) 454-3344 OFFICE

(505)454-0026 FAX

Dr. Phil Vergamini, MS P355

Office of University Research \& Science Educ.

Los Alamos National Laboratory

Los Alamos, NM 87545

(505)667-3628 OFFICE

(505)665-5596 FAX

Mr. F.T. Weaver

Oak Ridge National Laboratories

Martin Marietta Energy Systems

P.O. Box 2003

Oak Ridge, TN 37831

(615) $435-3207$ OFFICE

(615) 435 -
Mr. Jeffrey B. Weinrach, MS K572

Waste Minimization Program Office

Los Alamos National Lab

Los Alamos, NM 87545

(505)665-8292 OFFICE

(505)665-3390 FAX

Dr. Bobby Wilson

Texas Southern University

3100 Clebume

Houstion, TX 77004

(713)527-7133 OFFICE

(713)639-1999 FAX

Dr. Astatkie Zikarge

Director, Environmental Health Prog.

Texas Southem University

3100 Clebume

Houston, TX 77004

(713)527-7341 OFFICE

(713)639-1094 FAX 
NEW MEXICO HIGHLANDS UNIVERSITYILOS ALAMOS NATIONAL LABORATORY September 17-19, 1992, Las Vegas, NM

UNIVERSITIES, NATIONAL LABS, and AGENCIES attending WASTE MANAGEMENT TECHNICAL WORKSHOP: Research Partnerships at Minority Institutions funded by $U$. S. DOE

Alabama A\&M University

City College of New York

Howard University

Morehouse College

New Mexico Institute of Mining and Technology

New Mexico State University

North Carolina A\&T State University

Northern Arizona University

Rocky Mountain College

St. Augustine's College

Salish Kootenai College

Southern University and A\&M College

Texas Southern University

Tuskegee University

University of Turabo

Virginia State University

Los Alamos National Laboratory

Sandia National Laboratories

New Mexico Attorney General's Office

City of Las Vegas

U.S. Department of Energy

Office of Waste Management

U.S. Environmental Protection Agency

Martin Marietta Energy Systems, Inc

Renewable Energy Systems, Inc

Hazardous \& Radioactive Materials Bureau NMED 


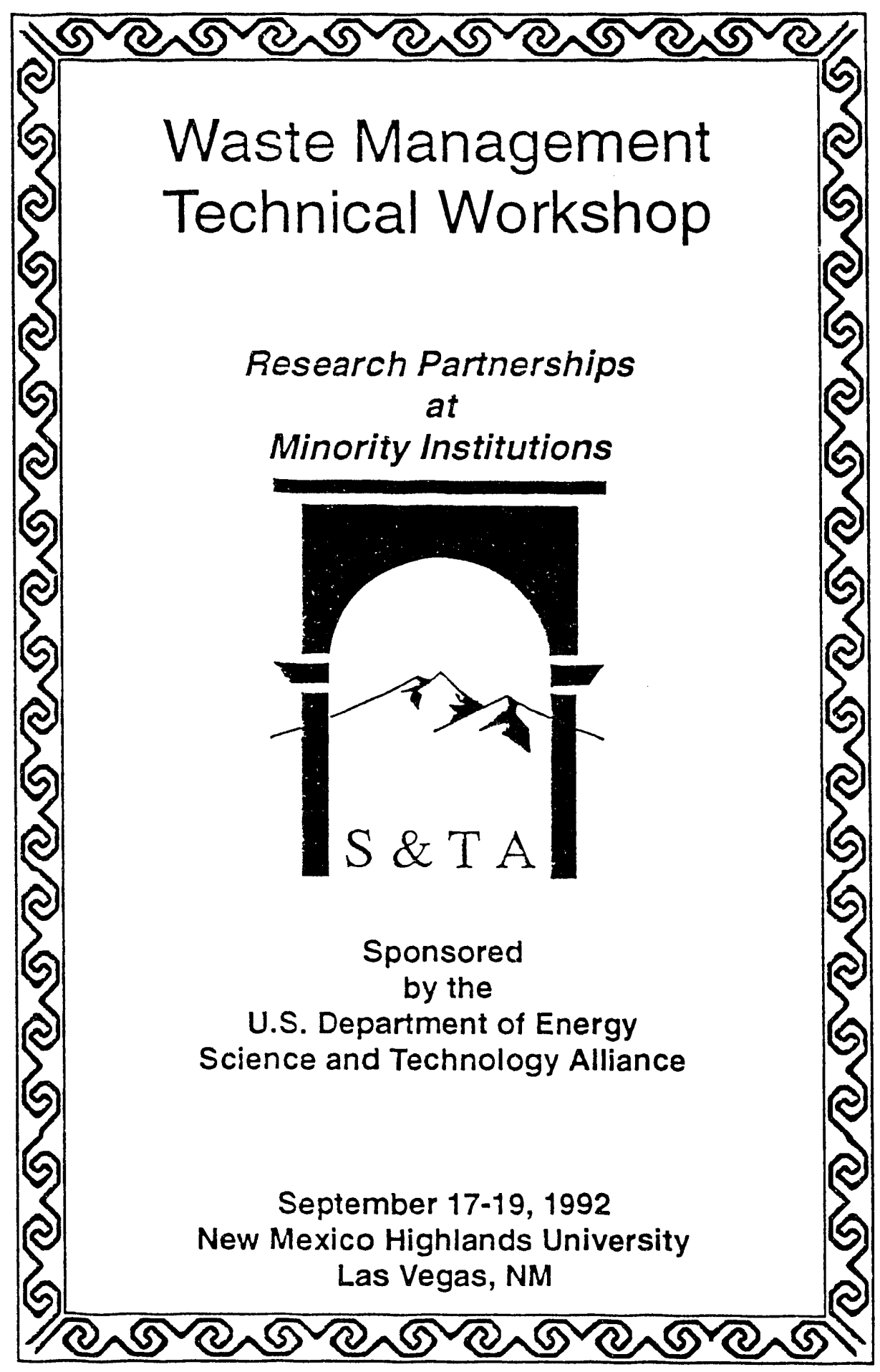




\section{WASTE MANAGEMENT TECHNICAL WORKSHOP Program}

\section{Thursday, September 17}

All events will take place in the NMHU Student Center Ballroom, unless otherwise noted.

8:00-9:00 Workshop Registration/ Continental Breakfast

9:00-9:20 Welcoming Remarks

Gilbert Sanchez, President, NMHU

David Sanchez, Deputy Associate Director

for Research and Education, LANL

Tony Martinez, Jr., Mayor, City of Las Vegas

9:20-10:15 Plenary Address

"Innovation in the DOE Waste Management

Program," Jill Lytle, Deputy Assistant Secretary, Office of Waste Management, L.S. Department of Energy

10:15-10:30 Break

\section{Presentations}

10:30-11:00 "Environmental Partnerships - the Key to Our Common Future," Jim Shipley, Program Director for Applied Environmental Technologies, LANL

11:00-11:30 "Energy, Environment and Technology Transfer at Sandia National Labs", \#1) Fred Norwood, Sr. Member of Technical staff, Sandia National Labs; $\$ 2$ ) Leo Gomea, Sr. Member Technlcal staff, Sandia National Labs.

Replacement for Jenny Haire 
11:30-12:00 "Potential Research Collaborations with the Environmental Protection Agency," Charles T. Mitchell, Environmental Health Scientist, Office of Research and Development, U.S. Environmental Protection Agency

12:00-12:15 Break

12:15-1:30 Lunch

Keynote Address: "WIPP and the State," Lindsay Lovejoy, Assistant Attorney General, State of NM

Presentations

1:45-2:15 "Community Waste-to-Energy Systems," James

Benson, President, Renewable Energy Systems, Inc.

2:15-2:45 "HBCU/MI Opportunity Areas," Terry Weaver, ERWM Minority Initiatives Program Manager, Martin Marietta Energy Systems

2:45-3:00 Break

3:00-3:20 "Solar Detoxification Project," William Dawes, Sandia National Laboratories

3:20-3:40 "WERC," Ron Bhada, Director, Waste Management Education and Research Consortium, New Mexico State University

3:40-4:00 "Environmental Education and Corporate Management: A Dangerous Gap," John Uribe, Associate Professor of Economics, NMHU

4:00 Announcements and Adjournment

5:00-7:00 Wine and Cheese Reception, The Plaza Hotel 


\section{Friday, September 18}

Workshop Theme: Creating collaborative research proposals between minority institutions and a) government, b) industry, and c) universities.

7:30-8:00 Continental Breakfast

8:00-8:15 Introduction of Topic I: "Getting Started." Phil Vergamini, LANL

8:15-9:30 Break-out group development of Topic I

9:30-10:15 Team reports and general discussion on Topic I. Moderator: Phil Vergamini, LANL

10:30 Buses depart New Mexico Highlands University for Los Alamos National Laboratory. Box lunches provided en route.

12:30-12:45 Buses arrive at Physics Division Auditorium, TA-3, SM-215

12:45-1:00 Welcome

1:00-1:30 Overview of waste management research efforts at LANL

1:30-3:30 Break-out groups tour selected facilities

3:30-5:30 Buses return to Bonita Ranch, outside Las Vegas

5:30-8:30 Barbecue and bluegrass music by The Atomic Grass

8:30-9:00 Buses return visitors to campus. NMHU vans will provide transportation to hotels. 


\section{Saturday, September 19}

7:30-8:00 Continental Breakfast

8:00-8:15 Introduction of Topic II: "Developing

Coalitions and Networking."

Abad Sandoval, LANL

8:15-9:30 Break-out group development of Topic II

9:30-10:15 Team reports and general discussion on Topic II Moderator: Abad Sandoval, LANL

10:15-10:30 Break

10:30-10:45 Introduction of Topic III: "Maintaining the Momentum."

Ken Holley, Sandia National Laboratories

10:45-12:00 Break-out group development of Topic III

12:00-1:15 Lunch

1:15-2:30 Team reports and general discussion on Topic III. Moderator: Ken Holley, Sandia National Laboratories

2:30-3:00 Round table discussion and summary of workshop Moderator: Melinda McClanahan, NMHU 


\section{Group Facilitators for Break-out Sessions}

Dr. Kenneth Bentson, Assistant Professor of Environmental Science, Department of Life Sciences, New Mexico Highlands University, Las Vegas, NM 87701

Dr. Harry Boston, Research Staff Scientist and Project Manager, Environmental Sciences Division, Oak Ridge National Laboratory, Oak Ridge, TN 37831

Dr. Milford Greene, Director of Engineering Programs, Morehouse College, Atlanta, GA 30314

Dr. Henry Hooper, Associate Vice President for Academic Affairs, Northern Arizona University, Flagstaff, AZ 86011

Dr. Jeanette Jones, Vice President for Research and Development, Alabama A \& M University, Normal, AL 35762

Dr. Bob Lessard, Professor of Earth Sciences, Department of Physical Sciences, New Mexico Highlands University, Las Vegas, NM 87701

Dr. Dilip Shah, Associate Professor, Department of Construction Management and Safety, North Carolina A \& T University, Greensboro, NC 27411

Dr. Jeffrey Weinrach, Staff Member, Waste Minimization Programs Office, Los Alamos National Laboratory, Los Alamos, NM 87545 


\section{Workshop Organization and Management Team}

\section{NMHU Director}

Melinda L. McClanahan, Dean, School of Science and Engineering, New Mexico Highlands University, Las Vegas, NM 87701

\section{LANL Workshop Coordinator}

Phil Vergamini, Staff Member, Office of University Research and

Science Education, Los Alamos National Laboratory, Los Alamos, NM 87545

\section{LANL S\&TA Coordinator}

A bad Sandoval, Scaff Member, Office of University Research and

Science Education, Los Alamos National Laboratory Los Alamos, NM 87545

\section{Logistic and Clerical Staff}

Cindy Griego, Program Secretary, HBCI/MI Environmental Technology and Waste Management Program; Administrative Assistant, Science and Technology Alliance, New Mexico Highlands University, Las Vegas, NM 87701

Clarice Garcia, Administrative Secretary, School of Science and Engineering, New Mexico Highiands University, Las Vegas, NM 87701

Benita Budd, Director of University Relations, New Mexico Highlands University, Las Vegas, NM 87701

Gwen Spatzi :r, Executive Director, Las Vegas Development Corporation, Las V-gas, NM 87701

Millie Saxman, Protocol Office, Los Alamos National Laboratory, Los Alamos, NM 87545

\section{Acknowledgements}

The v'orkshop team gratefully acknowledges the help and cooperation of Ms. Janice Odom, NMHU Interim Dean of Students; Mr. Toby Lucero, Student Center Operations Supervisor; the NMHU Environmental Science Club; Ms. Polly Mullen, NMHU Photographer; Dr. Drake Bingham, NMHU Department of Mass Communications: 'Nendy Quintana and Kody Simmons, Office of University Relations; the NMHU Print Shop staff; the NMHU Physical Plant; The Plaza Hotel; Steve Cathcart and Marcie Goke, Marriott Management Services; the S\&TA Ailiance members; and all others who helped make this workshop a success.

A special thank you and a rousing "Yee-haw!" to the Hooper family of Bonita Ranch. 


\section{Science and Technology Alliance}

The Science and Technology Alliance, funded by the U.S. Department of Energy, is dedicated to the enhancement of science and technology studies at minority universities. The Alliance's mission is to encourage and support minority students' involvement in research and education in scientific and technological fields through networking, research, and cooperative employment opportunities.

\section{Members}

New Mexico Highlands University

North Carolina A. \& T. State University

Sistema Universitario Ana G. Mendez

Los Alamos National Laboratory

Sandia National Laboratories

Oak Ridge National Laboratory

The Waste Management Technical Workshop

is hosted by

New Mexico Highlands University

and

Los Alamcs National Laboratory

ज्यक

Printed on recycled paper 
NMHU/LANL WASTE MANAGEMENT WORKSHOP:

RESEARCH PARTNERSHIPS AT MINORITY INSTITUTIONS

17-19 SE?TEMBER 92, LAS VEGAS, NEW MEXICO

\section{REPCRTSFRCM BREH.K.OUTGRCUFS:-3}

\section{GROUP 1}

\begin{tabular}{|c|c|}
\hline FACILITATOF:: & Cr. Aceeri Lessard \\
\hline PARTICIPANTE: & 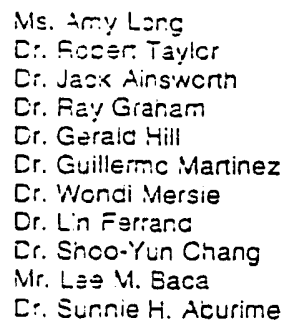 \\
\hline
\end{tabular}

1. Charac:erization ci hetercgenety of soil properties
A. Hycraulic proceries
B. Chemical prcestiles (crganicincrganic sorction)
C. Acolication to mixed wasies
D. Corretailen cerveen frcceries

II. Mcceling: use ci sir.ulaticn as a "accratory" for comclex systems

III. Cevelcpitesi nén iabifielc wethods
A. Paramererzarich
E. Monitoring
C. Testirg ci trestencal toceis

IV. Humar ceveicomen:

A. Inveivement di siucents (HS-Fho) in related research

$$
\text { GFCLP: Second Session }
$$

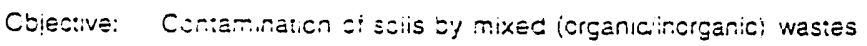

Goal: $\quad T=$ ceielcs cosi--itective inmeciation stiategles for scils coniaminaiec zy mixec ivastes.

Problems io acciess:

$$
\text { -..reiercgereics of schl projec:s }
$$

-...: ircm yesiercay's notes

Strategles: Incile tes serrouier, ecssibiy field compcnents

- Lesign las comconer: suci inat eacn insitition can

usetenrarce is zaricular excerise/faciities (invcivement of siucents at acciccr:a:e !evels:

:-_Ec analysis of sorcticnidescretion wastes on a variety of soil iypes

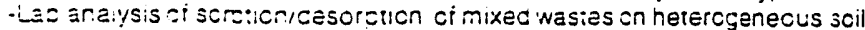
columns collectec itcmi the field

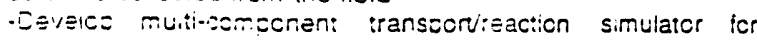
retercgerecus scils

- Use laz coitur cata io vertylcalibrate simulator

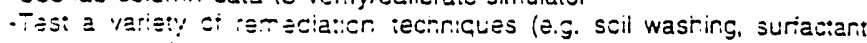
errancse scil vesnirc. sieam sitio mine) and soil columns centaminated cy tixez vasies

- ise simulaisr : $:$ ies: siraiegies on arciramy soils

\section{KEE=ING THE MONENTUM}

The soll arc grolecwa:er contamination anc treatment subgrcuo charge the

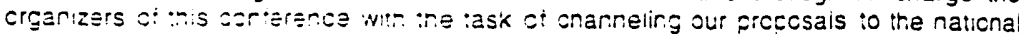
lacs anc kejerng suestele memcers nicrmed of the labcratcres' flans for utilization of cur zreceses. The natienal laes sircula feriorm critiques of the submitted frccosals frovicing ary cos:tive sinicisms anc rationale for negative feecback, aicng with suggestions tor improving ercecsals to make them suitable ior funding. 
FACILITATOR: Cr. Harry Boston

PARTICIPANTS: Dr. Wilter: Cdem

Dr. Joinn Rier

Cr. Guillermo Marinez

Cr. Ecgard Resto

Cr. Eiieen Chant

Mir. Ancy V:çii

Mr. Tairy Weaver

Mis. Earsara Lynon

Cr. Chuivwu Onu

Mr. Ed Aecischer

RESEAFCH PAOPCSAL TCPICS:

BICLOGICAL TRE.ATMENT:

Whole F!ant Sysiems/In Vitro Selection

In Situ Bicremeciation

Ex Situ Bicrivaiment

PRCCESミ MCOELING:

Mass Tiansier

GACLP 2. SECOND SESSION

HOW TO ELILO AND SUSTANIN COLLAECRATIVE RELATIONSHIFS

1. RESEAFCLH FARTICIFATICN CPPCRTUNITIES AT D.O.E. SITES

A. FACULTY

-Environm ental Venagement Career Occcrunuties tor

Miriorities

- Escuty Fesearon Paricicaticn

- Niriority Irstithiton Fesearco Travel

B. STJLENTE:G:aduate ar.c Undergraduate)

- SiLCer: Fesearon Panicicaticn

F:ziessicral Irierrisnics

- O. E. Ecence anc Engirearing Researcn Semester

- E.M.C.C.W. FE!lcinsnics (A.cacemic Year, Summer, at Lab)

2. FACFCSHL EEVE:CFMENT

A. Ecciccical Tieamer: (3)

- F-joess Mccetirg (:)

- Wasta ar Site Characierization (ङ)

3. CCALITICN SLILOINE

A. MCCE:SFOA LOINT PEOUECTE

- Lac-irvaraity jom: F-ojects

- University-University Fanitershics

-içr:iiicsicn anc Setection of Plants for Bicremediation (Metals anc
C:garics!

CHEMICAL CHAFACTEAIZATICNINASTE ANC SITE

Realoims Araivsis

Mernccs DEveicsmert:Extiacticn Characterization

In Situ EantersiCuntainmeni

SESSICH 3: FE:IEEIATICN FEZCNTAMIINATION TEOHNOLCGY

MAINTAININGTHE VICMENTUM

1) Imctern sr: wcrk:rg-evel zarinersnics berween minority instituticns and with cre

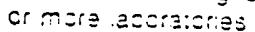
2) Euilc sc€cializec :tanirg comcorent(s) for facility and students into targeted

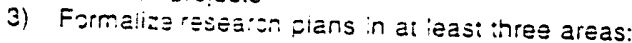

a) Sciar Ce:oxification

b) Eiciomicciaiich

c) Flan: ミニ Ec:es Vletacclisn of Wetals arc Crganıcs

4) Use esiablist:- LCE Fesearch-Paricioaticn programs to involve more faculty and siucents in sn-goirs orsjecis at laos

5) Ciate scec:al suminier scilazcraticns in iargetec areas 


\section{MUNIC!PAL WASTE MANAGEMENT}

WASTE MINIMIIZATION RECYCLING AND INDUSTRIAL ECOLOGY

\section{GAOUP 3}

FACILITATOR: Or. Jerirey Weinrach

PARTICIPANTS: Or. Jaya Krisnnagopalan

Dr. Ken Sccersirom

Cr. Merria: Helvensian

Ms. Crysiai Furiano

\section{GROUP 7}

FACILITATOR: Dr. Henty Hooper

PARTICIPANTS: Dr. Peter Eenscn

Dr. Rccer W. Taylor

Dr. Sr.cc-Yun Chang

I. Environmen:al Fecovery of Wastie Sites

A. Mine Wasies

B. Superiund/Ciear-ue Sites

1. Chemical waste sites

2. Radiation waste sites

11. Acprooriate use - meincds

A. Chafacierization of sites

i. Centrol sites

(a) Iriventery olants and arimals

(b) icentiry plants or iungi wnich act as bioacsumuiator 2. TEsi sites

(a) ic en:ificsticn of scminant scecies

(c) Siucy Frysiolcg\%, Eicchemisiry, Genetıcs, etc., of surviving scecies

(c) Determine accumulatcrs

1) inera coes acoumulation ccsur

2) how coes acoumulation ccoir

III. Possible Researcn :ccics / imetacle
A. Site Characterization - one year
B. Ceveicfment s: :ecrncicgy needed to clean up sites - iwo years
C. Comouter meceling of ecolcçical restoration
D. Commerc:aiizaicn of Tecnncicgy - iwo years

\section{Grouo 3 \& 7}

Three Picscsal Tcoics
i. Eypreduc: use
2. Me:al Secaraiticn
3. Fecycing aric erergy recovery frccesses

All iccics sthculd irriuce Sysioms Araiysis (mcceling)
Group 3 \& i: Ecology

Municipal Waste Management: Waste Minimizaticn, Recycling and Incusira:

Paricipants:

Or. Jett Weinrach - LANL

Dr. Henry Hoocer - Nonhern Arizona U.

Dr. Shoo-Yun Chang - North Carctina AZT State U.

Dr. Ken Soderstrom - University of Turaco

Dr. Peter Benscn - - Renewable Energy Sysiems, Inc.

Dr. Focer Taylcr - Alabama AaiM U.

Dr. Jaya Krisnnaģopalan - Tuskegee U.

Ms. Crystal Furiano - Stucent, New Mexico Highlands U.

TOPICITITLE

Wasie Treatment (ie. for the removal of heavy metals and hazardous orgarics: using piandcell sysiems with by-produci utilization.

OBJECTIVES:

The procosal will adcress waste treatment problems by utilizing suitable clantics! systems for waste (ie. sclid wastelland ill, waste water) ireatment. The scope will inciu $=\equiv$ the by-procuc: plant systems utilization techniques for energy, eic.

CURRENT STATUSI STATUS QUO:

Currently, there are sericus prcciems in the treatment techniques for wasie wa: from municipal sewage treatment, industrial processes etc. Some work has ceen carrie: out in the area of clant/cell systems utilization. but have nct seen very successiul. This projec: 'will aim to study known and new plant or cell sysiems ior waste water ireatment. Presenily, sucn an approach has not besn mace ior municipal soil waste ireaiment.

TASKS:

The majcr tasks in this project will include the followirg:

1. ICentiry specitic locai \& regional waste treatment problem(s)

Eg. Treating municical \& incustrial wastes

2. Icentify wncle clanvcell sysiemis) that are suitacle tor a sceciric waste ireaimen: picclem

3. Characterization of the wasis. Eitiect cf plent s'ssiem

$\therefore \quad$ Limits on iecinnoicgy

5. Sysism aralysis

6. Sy-crcduc: utilization to maximize rescurce recovery - reclamaticn : piarisicells - :esource (Energ\%, iesc sicck) :ecovery

7. Working mocels

LONG RANGE GOAL:

Data ga:nerea irom mocel was:e iteatment sysiems will ce used to cevelca an: engines piant sysiems for cther wasie reatment croclems. 


\title{
GROUP 4
}

FACILITATCR: Dr. Dilip T. Shan

Dr. Augustine Rios

PARTICIPANTS: Dr. Alan Gutjanr

Dr. Vasil Diyamangodlu

Mr. Frederico Asmar

Mr. Jim Kearns

TOPICS THE GROUP WOULD PURSUE:

SITE CHARACTERIZATION:

1. Hetercgeneity of site

2. Identify wnich parameters

3. Dynamic cnanges of site?

4. Site cnaracteristics? Beiore? Atter?

5. Impac: of regulationsistancards on methodology

6. Deveiccinglevolving meinces of characierization

\section{SAMPLING TECHNIQUES:}

1. What constitutes a representative sample?

2. What factors determine acpropriate analysis technique?

TOPICS TO BE PURSUED FURTHER BY THE GROUP

\author{
GRCUP 4, SECOND SESSION
}

DEVE:-CPING COALITIONS AND NETWORKING

1. PARAMETEFS

- Neec io icenity :he parameters, whether scil or watar, that will be most useful in soiving waste prodems asscciated with the site.

2. DYNAINIC CHANGES OF WASTE STCFAGE

- Realizing that wasiəs are not necessarlly stacle in terms of composition, there is a nees to silcy ire characier of the wasies. Studies neeced to I.D. the initial comccsition. of wastes. nciv they change over time, the rate of change, and if the changes proclce more hazarcous materiais.

3. SITE CHARACTEAISTICS OF SITE

- Meinccs usec. :ecrnicu三s acclied ard sarameters used would vary cepencing on whether a site is procesec or already in use. Questions required sucn as:

4. REGULATIONS

- Eticris needec to develcc site characteristic metrods and analysis which precuce corvir.cing, less zisoutae! e - esuits n 'ignt of changing iegulations. Prccose that we accress methodology issues from the shorter term stand point of meetir. reguiations so :hat ine longer :erm envirenmental heaith issues may be addressec.

5. METHCOS OF CHARACTERIZATICN

- Need :O icentify ire anaivicaliechniques that should be used defending on the sites. Veed :0 develop new :echniques of characterization so that new wasies are not gererated.

Ne's methcos needed for extraction of liquics that better simulate the scectic liquid in a faricular site.

\section{MAINTAININGTHE MCMENTUM}

THINGSTOCO:

1. Our Propcsal is Fミasicle

2. Work Srior: Term with Cre ci he Lacs

3. Pe-co the Procosai

4. Siek Fur.ding Agency 
FACILITATOR: Dr. Kennetn Bentson

PARTICIPANTS: Mr. Randall Leathers(NC A\&T State Univ.)

Dr. Astatkie Zikarge(Texas So. Univ)

Ms. Karla Garcia(NMHU)

Dr. Jaiara S. Turay(St. Augustine's)

Dr. Piara S.Gill(Tuskegee U.)

Dr. Stuart A. Nichoison(NMHU)

Dr. Chet Richmond(ORNL.MMES) crp@ornl.gov

Dr. Merritt Helvenstcn(NMHU)

TOPICS:

Bicmarkers

Datacases

Risk Communication

Tox. Chem.

IndustriavOccupational Hygiene

Environmental Transpert

Epidemiolcgy

Risk Assessment

Waste Sites:

Effects and exposure in humans

Page 1:

- Develco coalition for Fisk Assessment Methods Deveicpment and Technology Transter

-Procuce students trained in Risk Assessment Wethccs

-Community ecucation in Texicology

- Collaccrate with ctrer worksnco grcues:

(1) Exocsure Evaluaticn - direc:

a) mincriy communiry-traning siucents

b) clearilo workers

(2) Excosure Eraluation - Incirec:

a) environmental late siucies

c) Excosliz Estiria:Es sasec cn :ranspcri

(B) Epidermiclogy ci ex=csec zopliaticns

(4) Screening for heaitn efiecis near waste sites - vital stat:stics \& iumor regisires

(5) Problem of icxicolcgy ci comciex mixtures - lack of tesis

(6) Risk Estimaticn tecrnique development
a) comolex mixires
b) Risk communication

Pэgez:

(1) Cc:arn lisi of esiaclisnec hazarcous wasie sites

(a) Icentification of mincrity pcpulation in area

(3) Excosure Assessment :o cetermire if tevels are surficient to cause health proctems ai es:azlisn nazara materials zresent

(4) If ievels are estaclished. then epidemicicgical studies should pursue (need of epicis scecialisisi

a) gathering of heath iniormation frcm local heaith iactities, county, etc.

D) Local Surreysiccefulation snitis. eic.

(E) Ctiainirg esuits/ putlisni ecucaie community of results/ ceal with hears \& questions with incrksnces 
FACILITATOF: Cr. Milfors Greene (404) $\$ 25-6272$

PARTICIPANTS: NIs. Pat Hurley, (4C6) 675-48COX-344

Dr. E-nie Giacney, (EC2) $522-93333$

ils. Serese Aranna. (919) 373-9796 fim 5

Dr. Coris Garcia.

Or. E:nel Taylor, (S19) 334-7795

NCA \& $T$

1. Envircnmental Feccvery of Waste Sites

A. Mire Was:

B. Suferiunc/Ciean- o Sites

1. Chemicai wasiz sites

2. Raciaticn vaste sites

II. Appropriaie use - metrces

A. Charac:e: zaticn at sties

1. Corito sites

(a) in: $=n: c r$ glar:s and animais

(b) leeniary planits or fung; wnicn ac: as cioaccumulaicr

2. Tesi sites

(a) ICentiticaticn of ccminant scecies

(c) Silcy Physiolcsy. Biccnemistry, Genetics, etc., of surviving

szec:es

(c) De:ermine acoumula:crs

i) wners cces accumulation occur

2) how coes accumulation cccur

III. Possible Fesearch :coics : :imeracie

A. Sit: Charac:arza:ton - one year

E. CEvelccr $\cong r:$ ji : :crncicgy neeced io clean uo sites - two years

C. Comali:i noceling of Đecicgical resioration

D. Commerzalization si Tecrnciçy - wo years

Fesearch Tooics

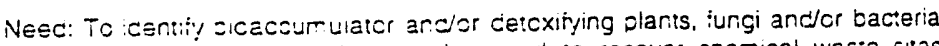
in a var:ey of actais wricr can be used io recover cnemical wasie sites througrcut the roritern nemiscriere.

Friicsecry s:き Frover srculd mimic ratural sucessicn as closely as zcssible. using naive or raturailzez jerial scecles innere fossicle

1. Site Chamas:=rza:ir

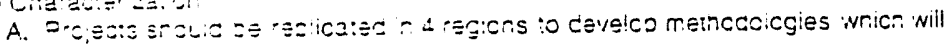
acsly in 3 an:

$\therefore$ Schir ess: 'Wart mesic raciiais

2. Sourvines: Warm. xerch hazia:s

3. Esuinnes: Coic. Xeric nazita:s

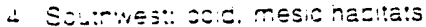

e. Gerere: LEscor

$\because$ Ez:Ezisn siugy sies. irciucirg a wasie site and a control site

2. leariny zomrar: so sc:es in each site, with an emcrasis on those scec:es wricn accear :0 ze survivirg in waste sites. Organisms chcsen

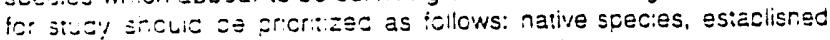
scec:Es, recenily ar nci-yet nirccucec species)

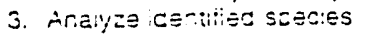

a. Cr.entcal analyses io cetermine if the crganisms are asumulating of g:Eaking down ioxins. Ienting spectic issices.

c. Genstic araiysis to compare site organisms with same $s=0 r:$ : t: $\mathrm{cm}$ cor:ivl site, and :0 icentity, if possicie scecific $\mathrm{G}=r$ Es : $=$ sconsicie : or suruval tratts.

c. Leie-mire growr zarameters of the organisms (metrco o

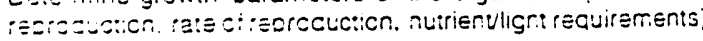

11. Ceveico Gigarisms as ziciecrnclcgical tocls

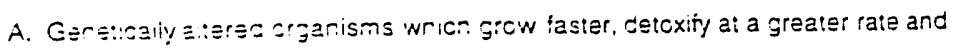
VCLUE

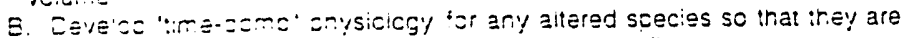

removez 'ig- :

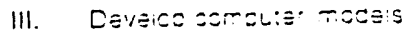

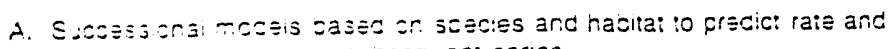
exzer:iz zranges zurng juesecueri series.

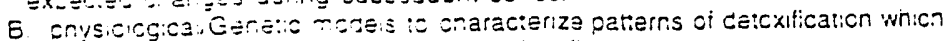

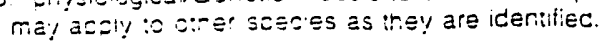


GROUP 6, Second Session

Nerwork Alternatives

1. Individual Regional Research

Send researchers to speciaity institutions to learn processes and standardize approaches

2. Share specimens, 'farming out' according to expertise (eg. ecol., genetics, physiol., comouter modelling.

3. Quality control mechanism (swap $\%$ of samples (espgenetic) to check reolaceability) This is a model for a joint-research project in restoration ecology. Many possibilities for specific research pulled from this model

Maintaining Momentum

1. What are $S$ sources?

Superiuno?

How do we commit with program directers and/or managers who have $\$$ (eg. division leaders)

2. Match interests with agencies. Via puclic meetings where face-to-face exchanges ocsur

3. Work with Ea. Succort organizations eg., NA, FEO, UNCE, AIHEC, LULAC

4. Match needs, pricrities, interests with trace organizations and individual industry (eg. chem.Man.Org)

5. Project Institutions

A. Develop/self. Assessment Instrument which identifies:

a. Personnel expenise, interests

b. Facilities

Sirengths

Needs

c. Insitutional commitment eg. release time, etc. available?

B. Quarerly update puclication io maintain progress, communication

IV. Develco commercial value ci recovery plants

A. Stacle seeclcining scurces

B. Growth and disinicution plans aiternative projec: Develop a successional

tecnnolcgy for site recovery--is it pcssible to accelerate succession?

SOLAR. CETCXIFICATICN MEETING

1. Program solit inio six activitiss.

2. UT. flat piate, centac: local indusiry

3. NMHU - modeling process periormance and eccnomics, solar troughs.

4. NC AZT - chemisiry of catalyst. contac: :ocal incustry.

5. Oak Ridce - analytical chemistry of solar detox (chemistry).

6. Los Alam.cs to suocort NMHU with computer mcdeling efforts (precess efficiency, economics)

7. S2EK irom each schcol as seen money.

8. All faculty memicers invoived in eaci schcol to come to Sandia. Selec: cate for mesing at Sandia (a meeting with NMHU facuity to precece date selectionl.

9. Eacn institution to make a list of equicment, prepare a bucget. selec: a zrincical invesilgator: this inicrmaticn will be due two weeks atter the meeting at Sancia.

10. Plan snould be for inree years (two as a minimum); also a representative each from LCs Alamos and Oak Ridge.

11. Projec: :0 emphasize stucent participation.

12. Proposal intenced ior EPA, DOE, NSF....

13. Coritac:s:

Shco-Yun Chang ( $(19) 334-7737,(919) \Xi 34-7 € 67$ FAX

Melinda McCianahan (505) 454-3528, (505) 454-31C3 fax

Abad Sandoval (EC5) 667-1230. (505) 665-3274 iemp. fax or (505) 665-5596.

Ed Aebischer (6:5) $574-7717,(615) 576-9496$ fax

Bill Dawes (505) 844--895 temp. (505) 845-8483 for messages, (505) 844-8884 fax iems 
MUNICIPAL WASTE MANAGEMENT

WASTE MINIMIZATION RECYCLING AND INDUSTRIAL ECOLOGY

GROUP 3

FACILITATOR: Dr. Jettrey Weinrach

PARTICIPANTS: Dr. Jaya Krishnagopalan

Dr. Ken Soderstrom

Dr. Merritt Helvensten

Ms. Crystal Furlano

GROUP 7

FACILITATOR: Dr. Henry Hocper

PARTICIPANTS: Dr. Peter Benson

Dr. Rocert W. Taylor

Dr. Shoo-Yun Chang

I. Environmental Recovery of Waste Sites

A. Mine Wastes

B. SuperfurddCiean-up Sites

1. Chemical waste sites

2. Faciation waste sites

I1. Appropriate use - methods

A. Characierization of sites

1. Conirol sites

(a) Inventory plarts and animals

(b) Identify plants or fungi which act as bioaccumulator

2. Tesi sites

(a) ICentification of dominant species

(b) Siudy Physiology, Biochemistry, Genetics, etc., of surviving

scecies

(c) Determine accumulatcrs

1) wnere does accumulation ocsur

2) how does accumulation occur

111. Possicle Fesearch tcpics / timetacle
A. Site Characterization - one year
8. Develocment of technolcgy needed to clean up sites - two years
C. Computer modeling of ecological restoration
D. Commercialization of Technology - two years

Group $3 \& 7$

Three Procosal Tocics

1. Eycrociuc: use

2. Meial Secara:icn

3. Fiecycting and energy recovery processes

All topics should inctuce Systems Analysis (modeling) 
Group 3 \& 7 :

Ecologicipal Waste Management; Wasie Minimization, Recycling and Industrial Ecology

Participants:

Dr. Jeff Weinrach - LANL

Dr. Henry Hoccer - Nonhern Arizona U.

Dr. Shoo-Yun Chang - North Carclina A\&T State U.

Dr. Ken Sodersirom - University of Turabo

Dr. Peter Benson - Renewable Energy Systems, Inc.

Dr. Robert Taylor - Alabama A\&MU.

Dr. Jaya Krishnagopalan - Tuskegea U.

Ms. Crystal Furlano - Student, New Mexico Highlands U.

TOPICITITLE:

Waste Treatment (ie. for the removal of heavy metals and hazarcous organics) using plant/cell systems with by-product utilizaticn.

OBJECTIVES:

The proposal will address waste treaiment prcblems by utilizing suitable plant/cell sysiems for waste (ie. solid waste/land fill. waste water) treatment. The scope will include the by-product plant systems utilization techniques for energy, etc.

\section{CURRENT STATUS: STATUS QUO.}

Currently, there are serious problems in the ireatment techniques for waste water fiom municipal sewage ireatment. industrial processes etc. Some work has been carried out in the area of clanv/cell systems utilization, but have not been very succsssful. This projec: will aim to study known and new piant or cell systems for waste water treatment. Presently, such an approach has not ceen made for municipal soll waste ireatment.

TASKS:

The major tasks in this project will incluce the following:

1. Icentity scecific locai a regicral wasie :reatment froblem(s)

Eg. Tieating municioal \& incusirial wasies

2. ICentity inole planveell sysiem; sl thai are suitable for

a scecific wasta treatment oroclem

3. Characierization of the wasie. effect of piant system

4. Limis on technclogy

5. Sysiem analysis

6. By-prscuct utilization to maximize resource recovery - reclamation of

7. piantsicells - resouice ienergy, ieed stock) recovery

7. Working mocais

LONG RANGE GCAL:

Data gatrered trom mctel waste ireatment sysiems will te used to develop and engineer plant systems for other waste treatment prociems. 
SOCIAL AND LEGAL ASPECTS OF WASTE MANAGEMENT

\section{GROUP 8}

FACILITATOR: Dr. Jeanerte Jones

PARTICIPANTS: Jonn Uribe

Anita Arcnibeque

Catherne Ratliff

Dorothy Leflore

Løo S. Gomez

Warren Miller

Bcboy Wilson

Ron Bhada

Armando C. Furlano

Charles Mitchell

PRCBLEMS: Identify social and legal aspects of waste management.

ASPECTS

Environmental Awareness

1. Ejucators

2. $K \cdot 12$

3. Scientists

4. non-scientists

5. Proiessionals

6. Regulators and bureaucrats (politicians)

RISKS

1. Health

2. Evcnomics

3. Logal

4 Sccial and esycnclcgical

5. Political

Planning/Implementation/Evaluation

1. Talking to sach other

2. Coniac: persons at each National Lab

3 . Capaoility siatements on each institution

4. Formation or working ieach (which may include persons outsice this group)

ミ. Detine rcle ci each gcvernmental agency and insttutional parner 


\section{SUMAARY OF RATINGS FROM 34 EVALUATION FORYS}

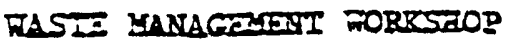

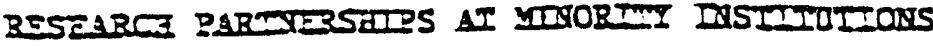

HOSTED BY

NEW MEKICO HIGEILANDS ONIVERSITY

AND

LOS ALAMOS NATIONAL LABORATORY

WORXSEOP ETALIATION

19 Seprember 92

For suture plawing porposes, please help us by giving your candid opirion abour eltis rorkshop. Tharik you for exting the time to anster the following:

1. overall trosicsiop

2. Presencations by DOE and EPA.

3. Presentacions by privare and corporace bustaess

4. Presencacions by nacional lavoracories

5. Smail goup activizies

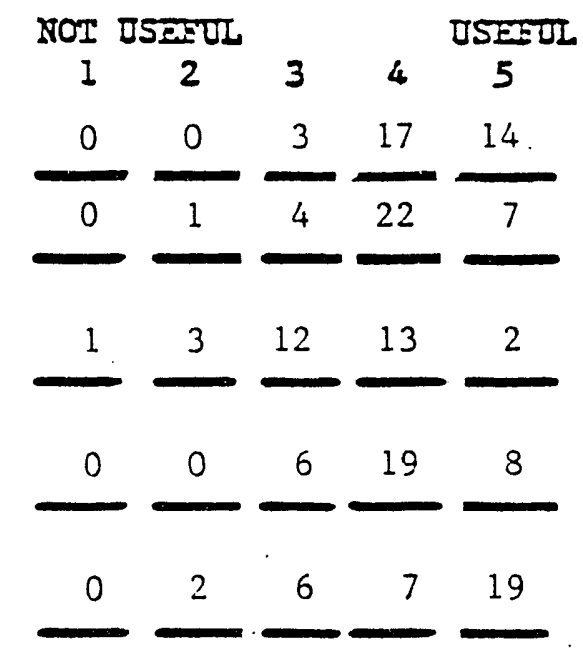

6. The concent of this rorksinop met मy expectations.

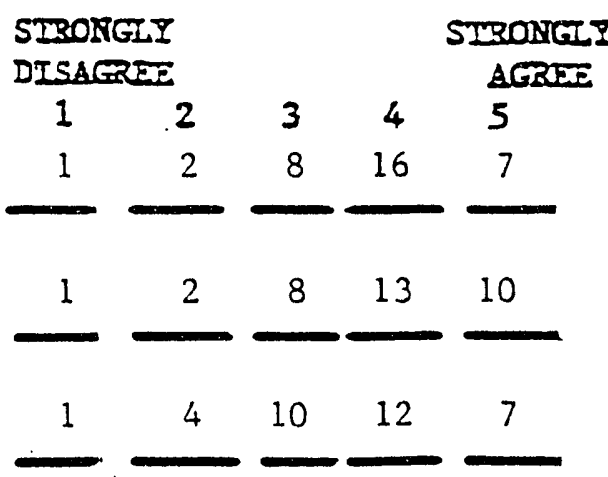

8. The techaical Ievel of the vorksiop ras appropriace for Iny reeds.

Whe best aspects of the roriksiop were:

The rorksiop could be improved by: 


\section{BEST ASPECTS OF WORKSHOP}

1. Meeting people in the S\&T Alliance and from other minority institutions (5 responses)

2. Personal contacts and networking; interaction with attendees; new collaborations and friendships ( 10 responses)

3. Meeting individuals with same research interests; getting to know people at other institutions and at national lab which will help me prepare a research project in my interests

4. Meeting other scientists from HBCU/MI and national laboratories

5. One-on-one interactions between faculty from different institutions

6. Good group comunication and sharing and translating ideas

7. Interrelationships between academia, private business, government, and communty

8. Information about projects at LANL and SN; obtaining information about technical work done currently and where funds are currently being spent

9. Visit to LANL (2 responses)

10. Information regarding matching my needs with DOE interests

11. Proposal development

12. Workshop break-outs; small group activities very productive (6 responses)

13. First day's individual presentations

14. Great organization of three-day meeting; workshop well organized and planned (5 responses)

15. Very nice social activities; nice mixture of activities planned; food was good; hospicality - outstanding ( 4 responses)

16. Above all, . the workshop was informative.

IMPROVEMENT POSSIBLE/SUGGESTIONS

1. Too many presentations on the first day; reduce number of formal presentations on first day and include one of the group activities that day (2 responses)

2. Use people frow national labs, EPA, DOE as facilltators for small groups; more funding agency participation in small groups; more active participation by labs and agencies ( 4 responses)

3. Invite scientists from labs to participate in each small group to provide expertise in discussion

4. Be more specific about the goals of the workshop before enlisting participants; set research agenda prior to meeting (2 responses)

5. Need more program directors represented from DOE, EPA, DOD, etc.

6. Provide specific guidelines for small group activities with more time for breakout groups

7. Target specific research funding possibilities or RFPs

8. More discussion on national Ráb needs

9. Make technical presentations on different projects of national labs; more technical information regarding ongoing research projects; more information on research work (3 responses)

10. Spending more time visiting the national lab and interacting with the research work being done and exchange our ideas with lab scientists; meet with upper level management at lab

11. The site visit to LANL was not very good - no content except two minor research projects

12. Include more industrial interests

13. Provide list of participants research interests

14. Evaluate each speaker individually to clearly see who should and who shouldn't be invited to speak at the next conference

15. Arrange a bus to bring people frow Albuquerque afrport instead of renting cars more cost effective; a bus service for a fee of $\$ 25$ or more can save the rest a lot of inconvenience ( 2 responses)

16. Need more time to soak up local culture and to shop for spouses 

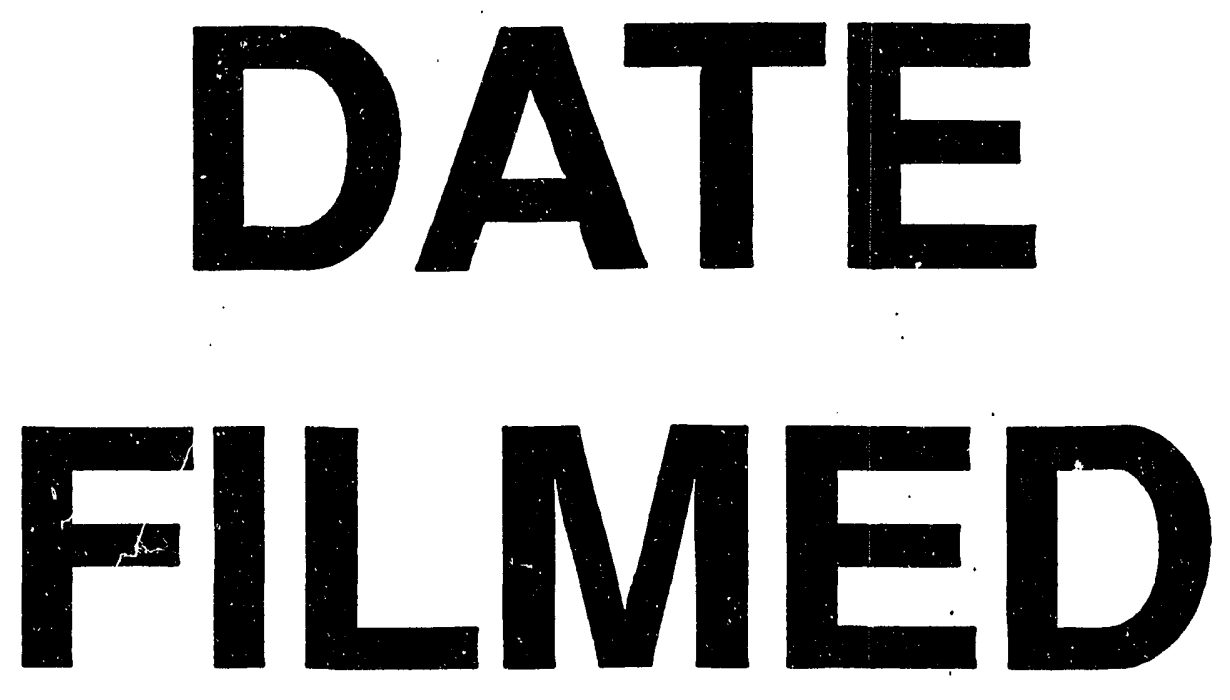

$10 / 13 / 93$
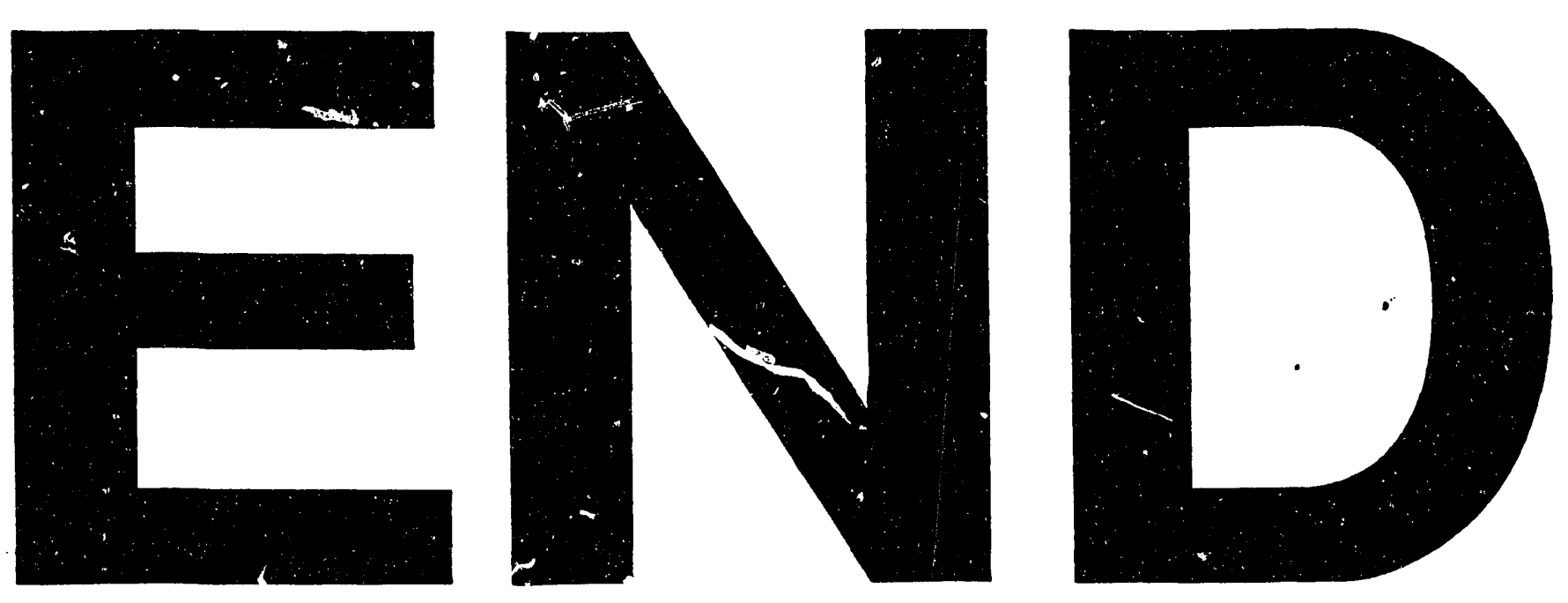
